\section{La Investigación-Acción como metodología para mejorar la práctica docente: tres casos en México}

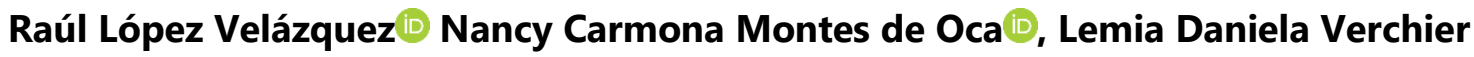 \\ Huizar ${ }^{\circledR}$, \\ Rebut: 04/06/2020 Acceptat: 20/07/2020
}

\begin{abstract}
Resumen
La investigación-acción se entiende como una forma de investigación centrada en realizar un cambio según las necesidades de los integrantes de un grupo social específico. En este artículo se describen tres intervenciones en la práctica docente de bachillerato basada en la investigación-acción. En la primera intervención se aborda el desarrollo de estrategias para fomentar la motivación en el aula. Dicha intervención se llevó a cabo estableciendo estrategias de fijación de metas desde la teoría de Edwin Locke, la cual afirma que la intención de alcanzar una meta es una fuente básica de motivación. Para la segunda intervención se implementó como estrategia el modelo basado en el análisis y discusión de casos con el objetivo de incrementar el interés y aprendizaje significativo, esto a causa de la identificación de la exposición de clases monótonas basadas en un modelo tradicional. La tercera intervención corresponde a un modelo de intervención en la enseñanza de ciencias de la salud, cuya finalidad es promover la motivación y despertar el interés del alumno y no sólo abordar temas propios de la ciencia, sino incidir positivamente en la construcción de nuevos conocimientos en el alumno de bachillerato con respecto a esta área. Los resultados más representativos en los alumnos fueron el desarrollo de habilidades cognitivas y socioemocionales, se destaca la mejora en la práctica del docente mediante la intervención en cada caso, dichos cambios fueron la enseñanza de manera efectiva y creativa, comunicación asertiva con los estudiantes y detección de las necesidades del contexto. Finalmente se concluye que a través de la metodología investigación-acción es posible elevar la calidad educativa a través de la mejora de la práctica docente
\end{abstract}

Palabras Clave: investigación-acción, educación, motivación, interés, estrategias.

\begin{abstract}
The action research is understood as a form of research focused on making a change according to the needs of the members of a specific social group. This article describes three interventions in high school teaching practice based on action research. The first intervention addresses the development of strategies to promote motivation in the classroom. This intervention was carried out establishing goal setting strategies based on Edwin Locke's theory, which states that the intention to reach a goal is a basic source of motivation. For the second intervention, the model based on the analysis and discussion of cases was implemented as a strategy with the objective of increasing interest and significant learning, this because of the identification of the exposure of monotonous classes based on a traditional model.
\end{abstract}


In the third intervention it corresponds to a model of intervention in the teaching of health sciences, whose purpose is to promote motivation and awaken the interest of the student by not only addressing issues of science, but positively influencing the construction of new knowledge in high school students with respect to this area. As main results we can mention the improvement in cognition and social skills, the improvement in teacher's practice is highlighted through the intervention in each case, these changes are represented by an effective and creative teaching, better communication between teacher and students and detection of context needs. Finally, we conclude that the action research is a methodology that helps to increase the educational quality through the improvement of teaching practice.

Keywords: action research, education, motivation, interest, strategies.

\section{Introducción}

La preocupación por la mejora educativa ha sido y sigue siendo prioritaria en muchos países, se habla de reformas, de implementación de modelos educativos innovadores, de metodologías, técnicas, de la creación de nuevas herramientas y materiales que ayuden al aprendiz a construir el conocimiento de una forma cada vez más facilitada. Lo cierto es que al considerar todo lo antes mencionado es de gran importancia tomar en cuenta a los dos protagonistas que intervienen en el proceso enseñanzaaprendizaje, el docente y el alumno quienes son los que interactúan en todo momento, una dicotomía constante difícil de concebir uno sin el otro.

Se reconoce la importancia central del profesor en el proceso educativo, debido a que es la figura que da forma y contenido a las propuestas educativas en el aula, como uno de los protagonistas del quehacer educativo, su acción se considera un indicador de cambio y por ende su contribución para mejorar la calidad en este ámbito es indiscutible. Esta preocupación surge debido a que las sociedades cambian y se desarrollan con el tiempo, es aquí en donde se originan distintas necesidades sociales y en donde la educación tiene que responder para cubrir algunas de ellas.

Sabemos que este proceso del que hablamos está influenciado por distintos factores en relación con las características propias del contexto en el que se desarrolla. En este sentido Martínez-Izaguirre, YánizÁlvarez, \& Villardón-Gallego (2017), menciona que vivimos en una sociedad que exige a un maestro cada vez más preparado para enseñar, generar un contacto con el conocimiento, así como situaciones de aprendizaje. La sociedad actual necesita a profesores que respondan eficazmente a las demandas del propio contexto, que acompañen y guíen al alumno en su aprendizaje, a un docente que aprenda a lo largo de la vida, se prepare continuamente, se forme, que genere nuevas preguntas, innove y aplique estrategias, a un profesional que investiga e integre procesos eficaces para su propia práctica.

La investigación-acción es una metodología que sirve para el propósito de mejora desde un punto de vista personal y autocrítica, en ella el docente puede encontrar oportunidad de mejora permanente. La adopción de esta metodología le permite al profesor encontrar orientación en aspectos pedagógicos, adquisición de competencias docentes, así como de conocimiento de su propia realidad, integración de información de su propio contexto, de procesos básicos del desarrollo profesional, conocimiento de la enseñanza y de su propia identidad, aspectos asociados a actividades significativas para la sociedad.

Así, la implementación de la investigación-acción en una situación educativa en particular, posibilita la identificación de oportunidades para mejorar las condiciones en que se desenvuelve el proceso de enseñanza-aprendizaje mediante la identificación de problemáticas específicas propias de la situación bajo análisis. 
Con las afirmaciones anteriores se menciona que el propósito central de este documento es dar a conocer desde tres diferentes intervenciones el uso de la investigación-acción como metodología para elevar la calidad educativa a través de la mejora en la práctica del docente y cómo puede ser aplicada en diferentes contextos.

\section{Investigación-Acción}

La investigación-acción es una metodología que se centra en llevar a cabo un cambio en un grupo social específico, siendo este cambio consecuencia de las necesidades externadas por los integrantes del grupo social en cuestión. Lewin en 1946 (citado por Latorre, 2004) describe a la investigación-acción como un ciclo de acción reflexiva que se compone de las siguientes etapas: planificación, acción y evaluación. Este ciclo comienza con una idea general en cuanto al tema sobre el que se elabora un plan de acción. Después, se hace un reconocimiento del plan y se lleva a cabo el primer paso de acción evaluando los resultados. Se revisa el plan general y se planifica el segundo paso de acción sobre la base del primero. Se realiza lo anterior en sucesivas ocasiones conforme lo requiera la intervención llevada a cabo. Al final, el proceso es evaluado en su conjunto. Estos pasos se ilustran en la Figura 1.

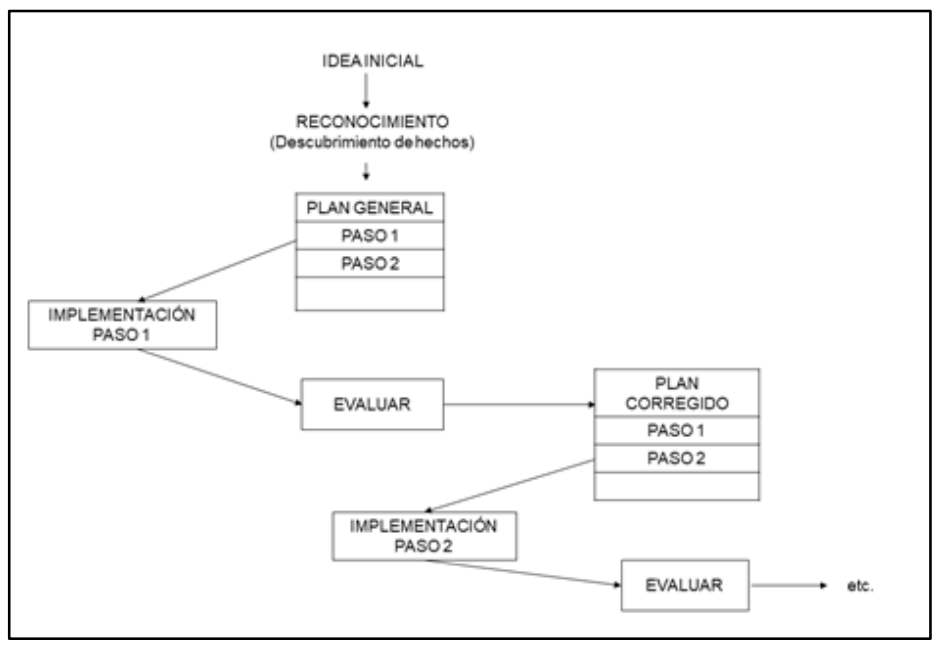

Figura 1. Modelo de investigación-acción de Lewin en 1946 (citado por Latorre, 2004).

En el marco de la educación, Elliott en 1981 (citado por Latorre, 2004) la entiende como una reflexión encaminada a modificar una situación basada en la comprensión de los problemas prácticos de los docentes. Por su parte, Carr y Kemmis en 1986 (citados por McKernan, 2001) la refieren como una forma de estudio autorreflexivo efectuado por quienes participan en situaciones sociales, con la finalidad de mejorar sus prácticas por medio de la comprensión de éstas dentro del contexto en que se llevan a cabo. Por otra parte, Navarrete y Farfán (2016) consideran que la investigación-acción en la educación permite describir actividades, tales como la planificación y mejora del currículum o el autodesarrollo profesional, que tienen en común el identificar estrategias de acción que son implementadas y que posteriormente se someten a observación, reflexión y cambio.

Cabe señalar que, según la postura de Carr y Kemmis en 1986 (citados por McKernan, 2001), quienes consideran a la reflexión como un elemento importante en la investigación-acción dentro del área educativa, ésta se vuelve una herramienta para poder llevar a cabo la modificación de situaciones que mejorarán el contexto en el aula.

De acuerdo con Elliott (2000), las técnicas y métodos que se pueden utilizar para recopilar la información necesaria en esta metodología son: diarios, perfiles, análisis de documentos, datos fotográficos, 
grabaciones, observadores externos, entrevistas, comentarios sobre la marcha, estudios de seguimiento, listas de comprobación, cuestionarios, inventarios, informes analíticos y la triangulación.

El análisis de la información así reunida es realizado en función de diversas relaciones que se manifiestan dentro de la práctica docente. Fierro, Fortoul y Rosas (2000) establecen la división de seis dimensiones de la práctica docente que sirven de base para dicho análisis: personal, interpersonal, social, institucional, didáctica y valoral. La implementación de estas seis dimensiones de la práctica docente en una situación educativa en particular posibilita la identificación de oportunidades para mejorar las condiciones en que se desenvuelve el proceso de enseñanza-aprendizaje mediante la identificación de problemáticas específicas propias de la situación bajo análisis.

- Dimensión personal: permiten reconocer al docente como un individuo con cualidades, características y dificultades, con ideales propios, motivos, intenciones, en donde su historia personal se vincula con su quehacer profesional.

- Dimensión interpersonal: las relaciones interpersonales que ocurren dentro de la escuela siempre son complejas (alumnos-docentes-autoridades escolares, padres de familia), estas pueden coadyuvar a eficientar procesos de aprendizaje.

- Dimensión Institucional: la institución escolar es el espacio en donde cada docente aporta sus intereses, habilidades, intenciones personales y saberes, siguiendo las tradiciones, costumbres, conductas y reglas marcadas desde la administración del sistema educativo que influyen en la labor del docente.

- Dimensión social: marcada por la diversidad de condiciones socioculturales, en esta dimensión se habla de las condiciones de vida tanto del alumno, del maestro y de sus familias, de las distintas oportunidades educativas, es aquí en donde el diálogo con el alumno se vuelve una parte fundamental para comprender sus necesidades educativas considerando vivienda, trabajo, salud, economía, recreación, costumbres, intereses.

- Dimensión didáctica: esta dimensión hace referencia al papel del maestro como agente que orienta, dirige, facilita y guía la interacción entre los alumnos y el saber, construyendo una experiencia enriquecedora.

- Dimensión valoral: las situaciones de enseñanza que el docente diseña dan cuenta de sus ideas, creencias, valores personales, actitudes, referentes teóricos, que se expresan en sus preferencias y juicios de valor, es así como el maestro de manera intencional o involuntaria comunica constantemente su manera de ver y entender el mundo, lo cual tiene gran trascendencia en la experiencia formativa de los alumnos.

Así mismo, las tres intervenciones presentadas se sitúan en las seis dimensiones anteriormente descritas: en la dimensión personal y la dimensión didáctica debido a que se interviene desde la práctica del docente, en la dimensión interpersonal que se relaciona a su vez con la dimensión institucional, dado que el espacio físico en el que se lleva a cabo la intervención es la escuela y se trabaja con el personal escolar como las autoridades y en ocasiones con los padres de familia en ciertas actividades a llevar a cabo y la relación más importante que es la que se tiene con los estudiantes, en cuanto a la dimensión social, tanto en el contexto de las escuelas privadas como de la pública, se encuentra una diversidad significativa en cuanto a las características de los estudiantes, desde el nivel socioeconómico como de la comunidad en la que viven, y la dimensión valoral, que al igual que la dimensión personal, es inherente a la práctica del docente y que se ve reflejada enteramente en su quehacer como guía. 
De acuerdo con Fierro et al. (2000) se describen 5 etapas de esta metodología, la primera hace referencia al análisis de la práctica docente, en ella se propicia la autorreflexión tomando en consideración las dimensiones que inciden sobre él mismo, la segunda etapa aborda la elección de la situación educativa que se desea transformar, la tercera se caracteriza por la búsqueda, aclaración y ampliación del conocimiento siempre en referencia a la propia práctica, la cuarta etapa permite confrontarse a esa situación que se quiere transformar, diseñando y aplicando un plan de intervención que lo permita, la etapa final corresponde a la recuperación de información a través del análisis de nuestro plan de intervención, es decir, se analiza el alcance que se logró con las acciones emprendidas para mejorar esa situación que se deseaba cambiar.

Según Elliott (2010) la investigación-acción le proporciona al docente facultades para la mejora de su práctica en el aula pues su objetivo es el de transformar, haciendo uso de esta metodología como herramienta que le permite generar conocimientos profesionales. Además, es un instrumento que permite la autoevaluación con el propósito de un desarrollo profesional.

El presente artículo describe tres casos sustentados en la metodología de la investigación-acción siguiendo con las fases anteriormente descritas aplicadas en la práctica del docente de bachillerato a partir de la identificación de distintas problemáticas, estas intervenciones tienen como propósito promover dicha metodología como una opción para fortalecer la calidad educativa enfocado en la mejora de la práctica docente en el aula y que pueda servir como propuesta para su aplicación en contextos similares.

La estructura de los casos se sintetiza en la descripción de un diagnóstico, correspondiente a la primera y segunda etapas de la investigación acción, la reflexión docente y la elección de la situación a cambiar, se describe también un modelo de intervención que corresponde a la tercera y cuarta etapas que se vinculan a la ampliación del conocimiento docente, diseño y aplicación de una intervención, por último, se explica la evaluación, etapa final de esta metodología.

\section{Caso No. 1: Implementación de estrategias de fijación de metas desde Locke para fomentar la motivación en el aula}

En cuanto a este primer caso de intervención, se optó por intervenir de una manera sistematizada a través de la metodología de la investigación-acción con el objetivo de mejorar la práctica del docente, debido a que se observaban diversos problemas dentro del aula, desde la indisciplina hasta el incumplimiento de tareas y apatía en la elaboración de las actividades en las clases impartidas.

Al utilizar esta metodología se proporcionaron elementos que fueron fundamentales para obtener resultados favorables en la modificación de la práctica docente, pues de acuerdo con sus etapas facilita la obtención de información utilizando diversos instrumentos que arrojan datos confiables. Posteriormente al analizar dichos datos, se pueden establecer acciones enfocadas en la práctica del docente, siendo éste el sujeto de acción en la intervención a realizar, las cuales son estructuradas de acuerdo con un modelo de intervención que nos ayudará a mejorar dicha práctica. Al final de esta intervención y a través de llevar a cabo una evaluación de los resultados podemos comprobar si el plan implementado fue funcional para lograr los objetivos buscados.

De esta forma el diagnóstico permitió definir que la problemática primordial a intervenir era en la falta de motivación de los estudiantes, para la que se indagaron diversas teorías de la motivación y se optó por utilizar como fundamento la teoría de fijación de metas de Edwin Locke y con ello, dar paso a la planeación de las clases a impartir a través de secuencias didácticas para posteriormente evaluar el modelo de intervención y conocer la funcionalidad de su implementación. Estas fases mencionadas se describen a continuación. 


\subsection{Diagnóstico}

El diagnóstico se llevó a cabo en la Universidad IUEM ubicada en el Municipio de Metepec, Estado de México, México con un grupo de 33 estudiantes del sexto semestre en la asignatura de Orientación Educativa, en la modalidad de bachillerato general universitario perteneciente al sistema de la Universidad Autónoma del Estado de México (UAEMex). Para la elaboración del diagnóstico se utilizaron dos instrumentos que permitieron la recopilación de información de la forma siguiente:

a) Diarios de clase (Anexo 1): Se llevó a cabo la aplicación de trece diarios de clase a los estudiantes, mismos que fueron contestados por el docente durante las trece clases impartidas respectivamente.

b) Grabación: Se llevó a cabo la grabación en audio de una clase muestra, misma que fue retroalimentada por una compañera de clase y por el asesor de la asignatura Intervención Educativa.

Después del análisis de la información recabada, se determinaron las siguientes categorías para identificar la problemática involucrada, como se muestra en la Cuadro 1.

Cuadro 1: Categorización de variables identificadas en el diagnóstico.

\begin{tabular}{llc}
\hline \multicolumn{1}{c}{ Transcripción } & Categoría \\
\hline - & La maestra se enoja mucho & Tolerancia a la frustración \\
- & Clase aburrida & Falta de motivación \\
Ejercicio aburrido & \\
- & Falta de atención & \\
Poca participación & Disciplina \\
- & Ambiente desordenado & \\
\hline & Compañeros desordenados & \\
\hline
\end{tabular}

De acuerdo con el análisis de los datos obtenidos, la problemática presente en el contexto donde se realizó la investigación consistió en la falta de motivación en los estudiantes, conduciendo a la interrogante de, si las estrategias de fijación de metas fomentarán la motivación en el aula para la mejora del manejo de grupo y la disciplina.

Con base en ello, la intervención se llevó a cabo en el grupo de 23 estudiantes de sexto semestre del bachillerato general en la unidad de aprendizaje Psicología II del Instituto Nicolás Guillén ubicada en el 
Municipio de Toluca, Estado de México, durante el período de febrero a junio de 2019. Su planeación fue definida a través de la estructuración de secuencias didácticas en las que, siguiendo con el fundamento de la Teoría de la Fijación de Metas según Edwin Locke, se busca fomentar la motivación en los estudiantes durante la impartición de la clase.

\subsection{Modelo de intervención}

Este modelo de intervención se sustenta en el fomento de la motivación en los estudiantes con el objetivo de dar respuesta a la pregunta de investigación ¿Las estrategias de fijación de metas fomentarán la motivación en el aula para la mejora del manejo de grupo y la disciplina?

La motivación forma parte importante en los ámbitos educativo y laboral, por ser un elemento esencial que conduce a la persona en lo que realiza y en cuanto a los objetivos hacia los que se dirige (Naranjo, 2009). Por otra parte, Mateo (2001) define a la motivación como un estado dinámico, el cual es deseable tanto para uno mismo como para los demás; estado en el que la mayoría de los motivos se llevan a cabo en un proceso cíclico definido por cuatro fases: Anticipación, activación y dirección, conducta activa y retroalimentación, y resultado. De este modo, la motivación se vuelve un aspecto siempre relevante para estudiar dentro del ámbito educativo (Bueno, 1993 citado por Mateo, 2001; Santrock, 2002 citado por Naranjo, 2009); siendo este último un pilar para que el estudiante se desenvuelva de forma óptima en el aula y logre el aprendizaje deseado, pues la motivación debe ser considerada como una disposición positiva para aprender y continuar haciéndolo de una manera autónoma por parte del estudiante.

\subsubsection{Teorías de la motivación}

\section{Pirámide de Maslow}

Maslow en 1954 establece una visión humanista de la motivación, destacando en las capacidades de la persona para lograr su crecimiento, sus características positivas y la libertad para elegir su propio destino (Ardila, 2001; Naranjo, 2009). Este presenta una jerarquización de las necesidades, que va desde las más fuertes hasta las más débiles. Para llegar a la siguiente escala de la jerarquía es importante satisfacer las necesidades de la anterior y así sucesivamente (Ardila, 2001).

Una vez satisfechas un escalón de necesidades, estas dejan de fungir como motivadores.

La teoría de Motivación-Higiene de Herzberg

Frederick Herzberg expuso su teoría de Motivación-Higiene orientada al trabajo y la gestión de empresas en 1959 en su publicación Motivación at Works, un informe de sus investigaciones y las de sus colaboradores acerca de la salud mental en la industria (Giovannone, 2011).

El análisis de las respuestas obtenidas permitió a Herzberg y a sus colaboradores concluir que la motivación en el trabajo se deriva de dos conjuntos de factores independientes y específicos:

Los primeros, asociados con los sentimientos negativos o de insatisfacción que los empleados aseguraban experimentar en sus trabajos y que atribuían al contexto de sus puestos de trabajo. Herzberg denominó a estos factores de higiene, porque actuaban de manera análoga a los principios de la higiene médica: eliminando o previniendo los peligros a la salud. (...) El segundo conjunto de factores se asociaba con las experiencias satisfactorias que los empleados experimentaban y que tendían a atribuir al contenido de sus puestos de trabajo. Herzberg denominó a este tipo de factores motivadores (Giovannone, 2011). 
Esta conceptualización de motivación considera dos factores principales: la satisfacción y la insatisfacción, mismos que determinan el desempeño del individuo según las condiciones extrínsecas.

Perspectivas conductual y cognitiva de la motivación

La perspectiva conductual señala que las recompensas externas (las cuales son eventos positivos o negativos que pueden motivar el comportamiento) y los castigos son centrales en la determinación de la motivación de las personas. El empleo de incentivos agrega interés y motivación a la conducta, así como dirigen la atención hacia comportamientos deseados y la alejan de aquellos que se considera inapropiados (Santrock, 2002 citado en Naranjo, 2009).

En cuanto a las teorías cognitivas Ajello (citado en Naranjo, 2009) menciona que estas hacen énfasis a que lo que la persona piensa sobre lo que puede suceder es de gran importancia para determinar lo que efectivamente ocurre.

Naranjo (2009) menciona que:

El sistema cognitivo es el que recibe y envía información a los otros sistemas: afectivo, comportamental y fisiológico, y regula el comportamiento de estos poniendo en marcha o inhibiendo ciertas respuestas en función del significado que le da a la información de que dispone. De esta forma, las ideas, creencias y opiniones que tenga la persona sobre sí y sobre sus habilidades determinan el tipo y la duración del esfuerzo que realiza y, por tanto, el resultado de sus acciones.

Para este enfoque, son los pensamientos los que guían la motivación del estudiante dentro del aula, enfatizándola en una motivación intrínseca.

Teoría de fijación de metas de Locke

De entre las teorías de la motivación existentes (Pirámide de Maslow, Motivación-Higiene de Herzberg, Perspectivas Conductual y Cognitiva, por nombrar algunas), la Teoría de la Fijación de Metas según Edwin Locke se considera que puede ser funcional para fomentar la motivación en los estudiantes. En los años sesenta, Edwin Locke presentó su Teoría de la Fijación de Metas u Objetivos, en la que afirmaba que la intención de alcanzar una meta u objetivo es una fuente básica de motivación (Locke, citado por Otero, s/a).

En este sentido, se entiende como meta todo aquello que una persona intenta alcanzar; es decir, la finalidad de una serie de acciones (Trechera, citado por Naranjo, 2009). Para que una meta u objetivo cumpla una función motivadora, debe contemplar los siguientes factores según Naranjo (2009):

El conocimiento; ya que se debe conocer la meta y los medios requeridos para alcanzarla.

La aceptación; debido a que debe existir acuerdo sobre lo que se desea realizar.

La dificultad; que significa que las metas deben ser difíciles, pero no imposibles; deben por tanto suponer un reto sin ser inalcanzables.

La especificidad; puesto que cuanto más concreto sea el objetivo, más fácil será aportar el esfuerzo necesario para lograrlo.

Al hacer la revisión de las teorías descritas anteriormente, se determinó que esta última es el modelo, de acuerdo con sus postulados, la que podría ofrecer aspectos funcionales en la práctica docente, tales como la implementación de establecer una meta al inicio de cada clase, establecido por el grupo y revisado al final de la clase para verificar que este se haya cumplido. Así, se podían observar resultados 
que ofrecieran información respecto al incremento de la motivación en los estudiantes no solo al revisar el logro de la meta, sino durante la clase en la que se observaría un incremento de la participación en las actividades por parte de estos.

Con base en este aspecto, las secuencias didácticas utilizadas para implementar el modelo de intervención se estructuraron de la siguiente manera: durante la apertura, se establece de manera grupal la meta a lograr, para continuar con el desarrollo de las actividades específicas del tema y finalizar en el cierre con la discusión acerca de la meta establecida. A continuación, en el Cuadro 2, se muestra el formato de secuencia didáctica que se diseñó, con base en la Teoría de la Fijación de Metas de Locke (citado por Otero, s/a):

Cuadro 2: Formato de secuencia didáctica diseñada para la intervención.

Objetivo: Duración de la clase:

Actividad previa: $\quad$ Competencias a desarrollar:

\begin{tabular}{|c|c|c|c|c|}
\hline \multicolumn{5}{|c|}{ APERTURA* } \\
\hline Actividad & Tiempo & Material & Actividad del docente & Actividad del estudiante \\
\hline \multirow[t]{3}{*}{$\begin{array}{l}\text { Establecimiento de la } \\
\text { meta de la clase. }\end{array}$} & $\begin{array}{l}5 \\
\text { minutos. }\end{array}$ & $\begin{array}{l}\text { Pizarrón. } \\
\text { Plumones. }\end{array}$ & $\begin{array}{l}\text { El docente hará mención del objetivo de la } \\
\text { clase y después anotará en el pizarrón la } \\
\text { meta establecida, en común acuerdo con el } \\
\text { grupo.** }\end{array}$ & $\begin{array}{l}\text { El estudiante redactará en } \\
\text { su libreta de trabajo la } \\
\text { meta establecida en grupo. }\end{array}$ \\
\hline & & $\begin{array}{l}\text { Libreta de } \\
\text { apuntes. }\end{array}$ & & \\
\hline & & Bolígrafo. & & \\
\hline
\end{tabular}

* Actividad a desarrollar previa a todas las clases.

** Características de la meta: especificidad, proximidad y dificultad.

DESARROLLO

Actividad

Tiempo

Material 
CIERRE

\begin{tabular}{|c|c|c|c|c|}
\hline Actividad & Tiempo & Material & $\begin{array}{l}\text { Actividad del } \\
\text { docente }\end{array}$ & $\begin{array}{l}\text { Actividad del } \\
\text { estudiante }\end{array}$ \\
\hline \multirow[t]{2}{*}{$\begin{array}{l}\text { Revisión del logro de } \\
\text { la meta establecida. }\end{array}$} & 10 minutos & $\begin{array}{l}\text { Pizarrón } \\
\text { Plumones } \\
\text { Libreta de apuntes }\end{array}$ & $\begin{array}{l}\text { El docente expondrá } \\
\text { la meta establecida al } \\
\text { inicio de la clase y } \\
\text { moderará la discusión } \\
\text { acerca de su logro. }\end{array}$ & $\begin{array}{l}\text { El estudiante } \\
\text { participará en la } \\
\text { discusión de si se } \\
\text { logró o no la meta } \\
\text { establecida y por qué. }\end{array}$ \\
\hline & & Bolígrafo & & \\
\hline
\end{tabular}

Evaluación del aprendizaje

Instrumento a utilizar

Evidencia

En cuanto a la evaluación del aprendizaje en la secuencia didáctica desarrollada se sustentó en el precepto de que la evaluación se convierte en un elemento imprescindible en la intervención educativa, debido a que proporciona la información pertinente para conocer el impacto y los logros que se obtuvieron en el grupo de estudiantes en que aquélla se implementó.

En la intervención realizada, los temas fueron: sensación y percepción, atención, memoria y aprendizaje, estilos de aprendizaje, inteligencias múltiples, interacción social, la dinámica de los grupos y su influencia en la conducta, estereotipos, teorías de la personalidad, factores biológicos, psicológicos y sociales que determinan diferentes rasgos de la persona, personalidad y conocimiento de sí mismo del plan curricular de la unidad de aprendizaje Psicología II.

\subsection{Evaluación}

Para la evaluación del modelo de intervención, se utilizaron los diarios de clase y una encuesta de satisfacción. Los primeros fueron aplicados a un estudiante al término del desarrollo de cada clase en donde se implementó el modelo de intervención, el cual se desarrolló en los diez temas referidos con anterioridad. En cuanto a la encuesta de satisfacción (Anexo 2) fue aplicada al término del semestre a los 23 estudiantes.

\subsection{Resultados}

Después del análisis de las evidencias recabadas, se determinaron los siguientes comentarios más significativos en los estudiantes:

- Los alumnos se sentían seguros de cumplir con el objetivo de la clase. 
- Al realizar las dinámicas, los alumnos se sentían motivados por aprender los temas vistos.

- El logro del objetivo los hacía sentir bien, emocionados, motivados e interesados en los siguientes temas.

- El objetivo planteado al inicio de la clase fue innovador (pues no se lleva a cabo en ninguna otra clase).

- La clase fue más dinámica.

- La clase fue más organizada/planeada.

- Buena participación de los estudiantes.

- El material utilizado fue suficiente para las actividades realizadas.

Con base a los comentarios antes mencionados, se puede destacar que la implementación de la secuencia didáctica basada en el modelo de intervención aplicado proporcionó resultados positivos que reflejan que en las clases implementadas los estudiantes se mostraron motivados en la realización de las actividades programadas, así como la evaluación refleja el logro del aprendizaje deseado. Otro punto del que vale la pena hacer mención, es que otro aspecto que mejoró fue la participación en equipo, el cual al inicio del curso se mostraba como una problemática para llevar a cabo las actividades, mismo que no era el objetivo del modelo de intervención pero que se vio influenciado de manera positiva.

Como área de oportunidad, se detectó que la disciplina sigue presentándose como un problema el cual puede abordarse desde la metodología de la investigación-acción, con la implementación de las fases aplicadas en el presente modelo de intervención.

\section{Caso No. 2: Aprendizaje basado en el análisis y discusión de casos ante el interés y el aprendizaje significativo}

\subsection{Diagnóstico}

La investigación-acción educativa es una metodología que permite no sólo detectar las problemáticas que los docentes presentan en el salón de clases, también sirve para diseñar actividades y estrategias en función de esa problemática, es una herramienta que promueve cambio social, además permite al docente tener autonomía y poder sobre la transformación de su práctica, acción que repercute en favor de los estudiantes y otros agentes de la comunidad educativa Latorre (2005).

Esta investigación tiene sustento en la metodología de la investigación-acción, la cual tiene como propósito profundizar la comprensión de la práctica cotidiana del docente, por lo cual adquiere una postura exploratoria, ya que se relaciona con los problemas prácticos experimentados por los docentes, en lugar de los problemas teóricos.

Esta investigación se desarrolló en el bachillerato "Instituto Cultural Nicolás Guillén" escuela particular incorporada a la Secretaría de Educación Pública, en Toluca, Estado de México, México, en el grupo de segundo año turno matutino en la Unidad de Aprendizaje de Mercadotecnia, con un promedio de 20 alumnos en clase. 
En la primera fase diagnóstica se recopiló información por medio de la aplicación de herramientas tales como videos, observación y retroalimentación de la clase por parte de un docente externo, diarios del alumno y diarios del docente, con la información recabada y analizada previamente junto con los docentes y compañeros de la Maestría en Práctica Docente se identificó el principal problema a atender, el cual era que el docente no promovía un ambiente de interés para los alumnos, esto debido a clases monótonas con ausencia de planeaciones, materiales y recursos didácticos, en consecuencia existía indisciplina y no se lograba el aprendizaje significativo en los alumnos.

\subsection{Modelo de intervención}

Por medio de la investigación-acción se selecciona el modelo de intervención óptimo para abatir la problemática que se identificó en la fase diagnóstico, tomando en cuenta la interrogante primordial ¿El aprendizaje basado en el análisis y discusión de casos como estrategia de enseñanza promueve el interés de los estudiantes para el desarrollo de aprendizajes significativos? y por medio de una investigación teórica exhaustiva se eligió trabajar con el Modelo de Aprendizaje Basado en el Análisis y Discusión de Casos por sus múltiples beneficios tales como los alumnos aprenden mejor tanto de forma grupal como individual, aceptan mayor responsabilidad en el desarrollo de la discusión, además fomenta la curiosidad y el desarrollo de destrezas que permiten el aprendizaje a largo plazo.

López y otros profesores en ITESM ( $\mathrm{s} / \mathrm{a}$ ) mencionan que mediante los estudios de caso los alumnos pueden desarrollar habilidades cognitivas como pensamiento crítico, análisis, síntesis, evaluación, aprendizaje de conceptos y aplicación de los mismos, habilidad para trabajar en grupo, comprensión de las necesidades del entorno, habilidades de autoexpresión, comunicación, aceptación, reflexión e integración, motivación por el aprendizaje y habilidad para la toma de decisiones.

El Aprendizaje Basado en el Análisis y Discusión de Casos es una estrategia la cual consiste en la descripción y posterior discusión sobre un hecho problemático procedente de la práctica profesional real que debe ser resuelto tomando como base los contenidos de la asignatura, que serán expuestos con anterioridad, así desarrollar sus habilidades (Trianes, 2012).

Este método se centra en el alumno como el actor principal y el docente tiene como función primordial ser agente de apoyo, orientador y facilitador de la información. Los casos permiten que se trabaje en conjunto los contenidos de diversas materias (Trianes, 2012).

[...] a partir de un caso real que se da en un contexto social (sea litio estratégico o no), el cual tiene implicaciones estructurales y colectivas, hay una situación problema donde el estudiante asume un rol e interactúa con otros estudiantes, no sólo en la identificación del problema sino también en la selección y sistematización de información pertinente, el planteamiento colaborativo de estrategias de solución y el análisis con profesionales de otras disciplinas. Ello implica que desarrolle actividades propias de su ejercicio como abogado (redactar, exponer, argumentar, etc.) busca proveer al estudiante de técnicas de análisis y ciertas destrezas profesionales que sirvan de medios para aprender de su propia experiencia, con una obligatoria reflexión sobre la responsabilidad social de su carrera (Torres, Iregui, \& Senior, 2015).

El aprendizaje activo es fundamental para crear un aprendizaje significativo, Torres et al. (2015) lo definen como "aquel en el cual todos los estudiantes son protagonistas de su proceso de aprendizaje, en experiencias que implican actividades más allá de escuchar y conlleva participar en simulaciones, discusiones, entrevistas, proyectos, análisis de casos, y lo más importante, reflexionar acerca del proceso"

Los mismos autores mencionan que "El aprendizaje activo se facilita con estrategias puntuales como el análisis de caso, que en este ámbito se da al estudiar y actuar desde las fases que debe trabajar el estudiante para actuar en cada etapa del proceso" 
Por las características de las materias ubicadas en la capacitación para el trabajo en administración, el aprendizaje basado en el análisis y discusión de casos permite al alumno un acercamiento a la realidad, esto quiere decir que los alumnos serán capaces de recibir la teoría y desarrollar habilidades a través de la resolución del caso, lo cual reafirmará y pondrá en práctica sus conocimientos.

Para alcanzar los aprendizajes esperados es conveniente tomar en cuenta el cono del aprendizaje de Edgar Dale (Véase figura 1) en el cual se puede identificar por medio de qué actividades se provoca el aprendizaje a largo plazo. Partiendo de este gráfico podemos decir que a través de los casos prácticos se participa activamente en la realización de la tarea haciendo un análisis, diseñando, creando y evaluando, según sea el caso, con ello se intenta inducir al alumno a un escenario lo más real posible, lo que en otros términos llamamos aprendizaje significativo.

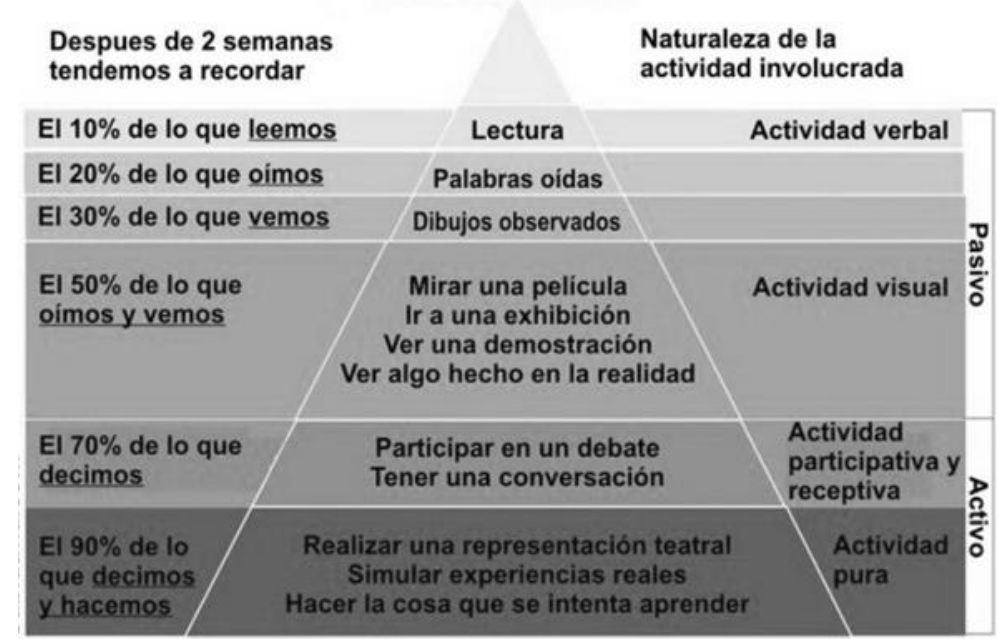

Figura 2. El cono de aprendizaje de Edgar Dale

El aprendizaje significativo se obtiene cuando los contenidos son relacionados con el aprendizaje previo del alumno de manera dosificada para que el alumno lo comprenda y pueda ser capaz de aplicar el nuevo conocimiento a su vida cotidiana (Ausbel, 1983).

Se realiza la aplicación de las secuencias didácticas basadas en el modelo de intervención, realizadas y estructuradas conforme a las necesidades de los alumnos y su contexto.

Para la elaboración de las secuencias didácticas se tomaron en cuenta los ejes transversales, las horas asignadas por semestre, los contenidos conceptuales, procedimentales y actitudinales, ya que son elementos que sirven de guía para cumplir los aprendizajes esperados.

A lo largo del curso se utilizaron diversas estrategias para la conformación de equipos para hacer partícipes a todos los alumnos, de tal manera que tuvieran distintas experiencias y se integrarán con el grupo.

Se analizó, discutió y resolvió un caso posterior a la exposición o investigación del tema, aunado a esto se aplicaron dinámicas de grupo, se trabajó con proyectos, se utilizaron materiales, recursos y estrategias didácticas.

\subsection{Evaluación}

La evaluación de la aplicación del modelo comprueba si se abatió la problemática identificada en la fase diagnóstico, además muestra los resultados positivos y negativos de la aplicación del modelo. 
"Esta parte del modelo nos permite una retroalimentación porque es aquí donde podemos juzgar los resultados" (Rodríguez, 2007, p. 11).

Se recabó información por medio de la aplicación de instrumentos tales como los diarios del alumno, diarios del docente, video de clase y la dinámica del "semáforo" esta última herramienta consiste en que los alumnos utilicen su creatividad al elaborar un semáforo en el cual puedan expresar libremente su perspectiva sobre la clase y el desempeño del docente, estos instrumentos se aplicaron en las últimas semanas del curso, con la información recabada se hizo una comparación y un análisis de los resultados esperados y los resultados obtenidos. también se tomaron en cuenta las dificultades y los distintos tipos de variables que se presentaron a lo largo de la investigación.

\subsection{Resultados}

Al trabajar con el aprendizaje activo a través de diversas estrategias como dinámicas, exposiciones, presentaciones, proyectos y sobre todo análisis de casos, los alumnos fueron capaces de aplicar los conocimientos y habilidades tales como trabajo colaborativo, síntesis, interpretación, creatividad, búsqueda de soluciones, toma de decisiones, entre otras, lo cual se convirtió en una actividad muy enriquecedora para el aprendizaje de los alumnos. En la Tabla 2 se muestran los resultados que generó el modelo de intervención.

Descripción de la Tabla 1. Resultados observados en los alumnos al implementar el modelo de intervención.

El éxito de los beneficios que ofrece el modelo de Aprendizaje Basado en el Análisis y Discusión de Casos depende en gran medida de las competencias que el docente manifieste en su elaboración, aplicación y dirección que les dé a las secuencias didácticas.

En la selección de los casos, es indispensable analizar el contenido previamente y verificar que cumpla con el objetivo, que su nivel de complejidad sea el adecuado para el grado escolar, que no sea excesivamente extenso y que sea lo mayor atractivo posible para el alumno, tomando en cuenta su contexto.

Existen algunos casos que se pueden complementar y/o nutrir con material audiovisual como videos, audios o infografía, lo que optimiza el aprendizaje en los alumnos.

Implementar el Aprendizaje Basado en el Análisis y Discusión de Casos aunado a otras actividades como proyectos, exposiciones y representaciones, propiciará la interacción del grupo y el interés en el alumno, de esta forma lograr los resultados académicos esperados.

\section{Caso No. 3: Modelo de intervención fundamentado en estrategias contextualizadas para la motivación en ciencias de la salud}

\subsection{Diagnóstico}

Esta última investigación se llevó a cabo en la Escuela Preparatoria Oficial No. 51 ubicada en el municipio San Mateo Atenco del Estado de México, México, institución educativa de bachillerato dependiente de la Secretaría de Educación Pública del Gobierno del Estado. La intervención del modelo se ejecutó con un grupo del tercer año del turno matutino conformado por 42 alumnos. 
Se utilizó la investigación-acción como una vía para mejorar la práctica docente, Navarrete y Farfán (2016) mencionan que esta metodología auxilia a mejorar cualquier proceso educativo tomando en consideración al docente como uno de los principales participantes en él, así como el rol que juega éste dentro del aula. Los mismos autores enfatizan la importancia de esta metodología en la formación de futuros docentes, así como en la profesionalización pedagógica de docentes con perfiles diferentes a la pedagogía o educación, ya que permite desarrollar la autocrítica y reflexión de su actuar docente, pero siempre en vías de encontrar un perfeccionamiento continuo y para beneficio del proceso educativo.

Debido a esta última idea se elige a la metodología de la investigación-acción para alcanzar el propósito de una mejora pedagógica desde un punto de vista personal y autocrítica, en la asignatura de Ciencias de la Salud. La adopción de la investigación-acción le permitió al profesor reconocer aspectos y áreas de oportunidad y encontrar esa orientación pedagógica necesaria partiendo del conocimiento de un estado inicial de enseñanza.

Para conocer ese estado inicial del proceso de enseñanza en Ciencias de la Salud se utilizaron instrumentos cualitativos (Fierro et al., 2000), al igual que en las dos investigaciones antes mencionadas, con el objetivo de recoger información que sirviera para identificar la principal problemática en la práctica del docente. De acuerdo con la metodología de investigación-acción, la fase de diagnosis apuntó a la necesidad de trabajar la promoción de la motivación en el alumnado para mejorar el proceso de enseñanza.

La videograbación de clase, la aplicación de diarios a alumnos y el propio diario del docente fueron los tres instrumentos que ayudaron a descubrir y describir lo que sucedía en clase de Ciencias de la Salud. Cabe señalar que bajo este principio Goetz y LeCompte (1984), mencionan que, para obtener y captar las definiciones y la realidad de los individuos de estudio, entendiendo a ésta como el resultado de una mezcla multifactorial, estos instrumentos permiten al investigador obtener información relevante y captar dicha realidad (véase anexo 1).

De esta forma se utilizó la observación participante y no participante para obtener información lo más objetivamente posible, es por ello que se llevó a cabo la triangulación y verificación de coincidencias desde diferentes perspectivas personal y la ajena al investigador con estos instrumentos. De esta forma se lograron identificar no sólo un problema (véase cuadro 3) que, desde la perspectiva epistemológica, es entendido como una situación a mejorar, en este caso desde la práctica docente o la del investigador (Latorre, 2005).

Cuadro 3: Categorización de las situaciones educativas de la práctica docente en la clase de Ciencias de la Salud.

\begin{tabular}{ccc}
\hline Falta de motivación & Escaso manejo de grupo & Indisciplina \\
\hline Ausencia de contextualización de & Docente no aplica medidas & Falta de orden y respeto cuando \\
los temas & correctivas & participan compañeros \\
Escaso dinamismo en la clase & Flexibilidad del docente & \\
Falta de estrategias para despertar & El docente pierde la fluidez de \\
el interés en la clase y motivar & la clase & \\
al alumno &
\end{tabular}


Distracción del alumno por

numerosos factores

Atención del alumno focalizada en

muchas otras situaciones

menos en la clase o temática

abordada por el profesor
El docente no monitorea las

actividades

Fierro et al. (2000) menciona que en la reflexión de la práctica docente se pueden presentar no solamente un problema en nuestra práctica, pueden denotarse varias situaciones en las que podamos mejorar, sin embargo, la comprensión y el trabajo de una situación educativa de forma particular requiere toda nuestra atención desde el abordaje, continuando con el tratamiento de la misma hasta la finalización y cierre como actividad de carácter cíclico.

\subsection{Modelo de intervención}

Debido a que la elección de la situación a mejorar obtenido del análisis de información en la etapa del diagnóstico fue la falta de promoción en la motivación en el alumno, se despliega la pregunta principal de esta investigación ¿Cuáles son las estrategias que se deben utilizar para fomentar la motivación tomando en cuenta las necesidades tanto del docente, del alumno y de la asignatura? Con la finalidad de darle respuesta a esta pregunta se llegó a la propuesta de considerar la contextualización de estrategias para facilitar la motivación.

Dicho esto, las estrategias pedagógicas contemplan tanto las estrategias de aprendizaje como las estrategias de enseñanza, las primeras ayudan al alumno a alcanzar los conocimientos a través del desarrollo de habilidades que le auxilien en asimilar y comprender mejor la información a partir de su análisis, a aprender y poder así solucionar problemas; las segundas se refieren a la diversidad de caminos $\mathrm{u}$ opciones que el docente puede tomar para presentar los contenidos de la asignatura para llegar a crear el conocimiento y comprensión de la realidad en el alumno (Díaz y Hernández, 1999).

Los fundamentos teóricos auxiliaron para hacer una aproximación al modelo pedagógico, Salmerón (2013) menciona que uno de los objetivos primordiales de la educación formal debe ser la transferencia de los aprendizajes al entorno en el que el alumno se desenvuelve y comparte con otros, es decir, la aplicación del conocimiento adquirido a su propia realidad y fuera del aula.

Se planteó la contextualización de estrategias educativas en la enseñanza de las Ciencias de la Salud, estrategias que con la estimulación de experiencias le permiten al alumno el acercamiento con su propio contexto, en donde se le permite ser agente activo y participativo, alejándolo de la rutinaria y ortodoxa praxis docente, incluyéndolo en actividades auténticas que ayuden a construir un aprendizaje significativo. De esta manera se motiva al alumno, se despierta su interés por resolver problemas de la vida cotidiana, comprenderlos y explicarlos, se le conduce a reestructurar preconcepciones, alcanzar competencias, modificar hábitos, reflexionar, y tomar conciencia del beneficio del conocimiento científico (Flores, 2013; Rioseco y Romero, 2000).

Es interesante ver a la enseñanza de la ciencia de forma contextualizada desde el punto de vista de Busquets, Silva, \& Larrosa (2016), pues en su escrito "Reflexiones sobre el aprendizaje de las ciencias naturales. Nuevas aproximaciones y desafíos" mencionan que la falta de motivación puede vincularse a la poca contextualización de los temas con la vida cotidiana de los alumnos a través de ejemplos o 
fenómenos cercanos. Esta motivación y estimulación dependen de la experimentación y de la dinámica de las actividades que permitan al estudiante lograr aprendizajes significativos.

Las estrategias utilizadas para el modelo de intervención fueron el estudio de casos, la práctica de laboratorio, dramatización y aprendizaje basado en proyectos (ABPr) debido a que cumplen el requisito de poder ser adaptables y facilitar la aproximación al contexto y las necesidades de aprendizaje además para comprobar los beneficios de cada estrategia de acuerdo a los teóricos como el incremento de la motivación e interés, desarrollo de habilidades sociales y comunicativas, facilitan el alcance de las competencias, la toma de decisiones, el pensamiento crítico, la transferencia de conocimientos, la mejora del aprovechamiento, entre muchas otras (Balongo y Mérida, 2016; Cardona, 2013; López, Ramos, Pato, \& López, 2012; Orkaizagirre, Amezcua, Huércanos, \& Arroyo, 2014).

Posterior a la fase de ampliación del conocimiento respecto a la conceptualización y la teoría se desarrolló un plan correspondiente a la fase operativa del modelo, en donde se incluyen el estudio de casos, la práctica de laboratorio, la dramatización y ABPr como estrategias adaptadas para actividades relacionadas con los temas incluidos dentro del programa de Ciencias de la Salud con la finalidad de promover la motivación en el alumnado. Dicho plan incluía el uso de diversos recursos y medios didácticos (libros, revistas, muñeco de primeros auxilios, botiquín, videos, presentaciones, lecturas, ruleta) y espacios escolares (biblioteca, laboratorio, auditorio, aula, sala de cómputo) para auxiliar el aprendizaje.

\subsection{Evaluación}

Durante la etapa operativa del modelo se aplicaron de nueva cuenta los instrumentos de recogida de información el video, el diario del alumno y del docente con la finalidad de registrar todos los pormenores posibles para su posterior análisis en la fase de evaluación. Tanto los diarios del docente como los del alumno, se elaboraron y aplicaron respectivamente, después de agotada cada sesión y con cada estrategia ejecutada, posteriormente se comprobaron detalles que denotaron el cambio en comparación con el estado inicial. El análisis de estos instrumentos de nueva cuenta se realizó lo más objetivamente posible considerando el punto de vista del alumno como actor participante en el proceso, el del docente investigador y de la videograbación como observación no participante y con el punto de vista de otros compañeros docentes.

\subsection{Resultados}

Entre los puntos de cambio en relación con la situación educativa elegida para transformar, se denota el cambio significativo en el rol del docente, y la incorporación del alumno como un elemento proactivo y no como una figura abstracta en el proceso de enseñanza aprendizaje y el uso de diversos espacios dentro del centro educativo, dos factores intrínsecos dentro de la planeación de la intervención que permitieron el alcance del objetivo. La práctica docente se mejoró encontrando diferencias significativas y contrastando el estado inicial con el estado final de dicha práctica (véase cuadro 4). Al mismo tiempo se constataron los múltiples beneficios de acuerdo con los autores consultados además de cubrir la promoción de la motivación con las estrategias utilizadas. Dentro de los beneficios verificados de acuerdo con los teóricos se comprobó:

- El aumento de la motivación e inclusión participativa en el alumnado en cada actividad con estrategias contextualizadas

- La contextualización mejora la transferencia de los conocimientos, estos se aplican en un ámbito distinto al escolar

- Mayores relaciones interpersonales, cooperación y colaboración 
- Mejora del ambiente o entorno de clase

- Desarrollo de habilidades sociocomunicativas

- Mayor alcance de competencias disciplinares en ciencias de la salud

- Generación de mentores emocionales entre pares académicos

Cuadro 4. Comparación del antes y un después con referencia a la situación educativa elegida

\section{Identificación de incidencias en la situación educativa \\ elegida en la fase \\ diagnóstica}

Identificación de incidencias en la situación educativa elegida en la fase evaluativa
El profesor no motiva al alumno
El profesor funge como promotor de la motivación, alienta al alumno a profundizar temas mediante la interrogación, estímulos el interés mediante ejemplos sencillos que al alumno

le interese aprender, es un guía emocional, comparte experiencias y ejemplos reales, proporciona actividades y ejercicios alcanzables, promueve la socialización y la internalización del conocimiento.
La actitud del docente es pasiva

Las estrategias que desarrolla el docente, no tienen relación con el contexto del alumno

El docente no desarrolla estrategias atractivas para generar motivación de los alumnos y despertar su interés

Ausencia de dinamismo en la clase

Las clases son monótonas y tediosas generalmente

Clases poco ilustrativas
El profesor se desenvuelve según lo recomendado en cada estrategia implementada de forma tal que el alumno sea el principal actor, sin embargo, su papel ya no es pasivo, ahora direcciona al alumno de forma más cercana (le conoce, pregunta sus inquietudes, observa su actuar, su interés) con la finalidad de apoyar su aprendizaje, promueve el conocimiento científico a la comunidad estudiantil, provee de ejemplos suficientes de la vida cotidiana para motivar al alumno.

El discente participa proactivamente en las actividades, muestra interés, pregunta constantemente, se acerca al docente para aclarar dudas, proporciona experiencias propias o familiares para ampliar y profundizar el tema, coopera en equipos con sus compañeros, se emociona ante una nueva actividad, emite comentarios constructivos y positivos, a la clase y hacia el docente, fomenta el debate y la discusión, comparte información y su punto de vista, analiza e interioriza la información para aplicarla en su contexto. 


\section{Discusión}

La investigación-acción se entiende como una forma de investigación que se centra en realizar un cambio, resultado de las necesidades externadas de los integrantes de un grupo social específico, en este caso, en un aula educativa. Por ello es importante tomar en cuenta diversos elementos que se presentan en este contexto, que van desde problemáticas presentadas por los estudiantes, hasta la práctica del docente y la forma en la que esta influye en los alumnos, siendo este último el aspecto que se puede modificar para mejorar la dinámica en el aula y mejorar el aprendizaje.

Según Rodríguez (2016) "El salón de clases es un espacio afectado por una multitud de factores intrínsecos y extrínsecos que pueden facilitar u obstaculizar el aprendizaje." Por ello, la investigaciónacción proporciona al investigador las herramientas para identificar los aspectos que influyen de manera significativa en el aula, siendo algunos de estos los componentes principales a modificar para mejorar la práctica del docente y promover el aprendizaje en los estudiantes.

Se destaca que la metodología de la investigación-acción educativa ha servido en diversas investigaciones como principal medio de transformación (Quintero, Zuluaga, \& López, 2003; García, 2016; Rodríguez 2016) cuyos resultados resaltan clases más eficientes, mejora de la comunicación, estudiantes motivados a aprender, el cual era el objetivo del cambio de la práctica del docente.

Es así que en los tres casos en los que se aplicó la investigación-acción se identificaron mejoras en la práctica del docente, así como el desempeño y aprendizaje de los estudiantes. Este beneficio es de notable mención por Martínez-Izaguirre et al. (2017) asegurando que para ello es necesario contar con profesores capaces de responder eficazmente a las necesidades del contexto educativo, con deseos de una preparación constante, de seguir innovando y generando nuevas preguntas para seguir avanzando en la educación, así mismo, de ser capaces de analizar con evidencias y la aplicación de instrumentos y estrategias cuya validez ha sido comprobada pero que al mismo tiempo se posibilite su adaptación a los diferentes contextos.

En cuanto a la reflexión sobre la práctica del docente, es una clara oportunidad para introducir mejoras en el entorno educativo de manera que dichas modificaciones se hacen de forma intencionada pero no sin antes conocer el estado actual que gobierna cada clase y considerando las situaciones como productos multifactoriales del contexto y de los propios actores principales en él (docente-alumno). Esto permite aclarar el papel en nuestra forma de enseñar, la forma en que aprenden nuestros alumnos, 
sus intereses y motivaciones, el porqué de sus errores y de forma similar permiten reestructurar actividades que no cumplen con el objetivo para el cual se crean (Flores, 2013).

Bajo esta misma idea, Busquets et al. (2016) menciona que es necesario una constante reflexión y autoevaluación del quehacer docente e implementar actividades para mejorar en el aula. Ellos brindan una mirada integral al papel que juega el docente en el proceso de enseñanza-aprendizaje para contrarrestar las múltiples dificultades que se pueden presentar en él.

Es de suma importancia mencionar que las diferentes dimensiones de la investigación-acción (personal, interpersonal, institucional, social, didáctica y valoral) enmarcan el actuar del docente. Se propone consideran estas 6 dimensiones para poder generar un impacto positivo para los diferentes contextos en el que se ejecuta. Para cada caso aquí presentado se consideraron las limitantes dentro de cada dimensión como el reconocimiento de las capacidades del docente, permisos y política institucional, material y espacios disponibles para la intervención, recursos didácticos, impacto en la sociedad con las acciones ejecutadas desde el aula y las relaciones entre miembros de la comunidad de aprendizaje (Fierro et al., 2000)

Tomando como referencia que nos encontramos en un espiral en el cual buscamos mejorar continuamente nuestra práctica docente, hacemos uso tanto de la reflexión continua como de la información que nos proporciona la evaluación para descubrir qué otras problemáticas han surgido e iniciar de nuevo el ciclo a partir de un nuevo plan de intervención.

En cuanto a similitudes que encontramos en los tres casos descritos, el principal es que al implementar la investigación-acción como metodología para mejorar la práctica docente en diversas problemáticas, es significativamente funcional para lograr cambios en esta y propiciar el aprendizaje en los estudiantes. Otro aspecto que se muestra en común es que la motivación es un factor fundamental para propiciar el aprendizaje, si bien en el segundo caso no se determinó como la problemática primordial a intervenir, los resultados en el diagnóstico la resaltan como relevante.

Entre las diferencias que encontramos es que el contexto de cada aula es diverso y por ello, aunque se trate de una misma problemática, como en el primer y tercer caso, la forma de intervención dependerá de distintos aspectos como son los recursos tecnológicos, las normas institucionales y la habilidad del docente para aplicar y dirigir el modelo de intervención

\section{Conclusiones}

A partir de la revisión de los resultados obtenidos en cada una de las intervenciones descritas, se puede concluir en primera instancia que la metodología de la investigación-acción funge como una opción para fortalecer la calidad educativa enfocada en la mejora de la práctica docente en diversos contextos. En segunda, la investigación-acción es una metodología efectiva para abordar problemáticas en el proceso de enseñanza-aprendizaje, permitiendo generar propuestas de mejora para el docente y los estudiantes.

Por otro lado, las teorías de la motivación existentes ubican a ésta como un factor determinante para encauzar el aprendizaje de los alumnos siendo de gran relevancia que el docente sea capaz de guiar, motivar, despertar el interés y retroalimentar a los alumnos antes, durante y posterior a su aprendizaje.

Así mismo para lograr un aprendizaje significativo en el alumno se requiere de docentes capacitados que no sólo expongan un tema, sino que se involucren en la implementación de estrategias y recursos didácticos necesarios para hacer más sencilla la adquisición de conocimientos y habilidades que le sean 
útiles y aplicables en su vida personal, académica y profesional. De igual manera es imprescindible conocer las distintas herramientas y estrategias didácticas y saber en qué momento aplicarlas para facilitar y potencializar la enseñanza considerando el contexto en el que se ejecutan, así como regularlas y dosificarlas para hacer ajustes durante y posterior a su aplicación.

Es fundamental la evaluación de los resultados obtenidos para realizar las modificaciones pertinentes y sustentar la toma de decisiones a fin de transformar la práctica docente.

Es conveniente continuar con la ponderación de las acciones definidas en intervenciones posteriores, como parte de la investigación-acción, con el objetivo de afinar sucesivamente el proceso con lo que se tenga plena certeza de haber resuelto la problemática identificada de manera significativa.

\section{Referencias bibliográficas}

Ardila, R. (2001) Psicología del aprendizaje. México: Siglo XXI Editores. Recuperado de: https://books.google.com.mx/books?id=aZ1DHwytQO8C\&pg=PA81\&dq=definici\%C3\%B3n+d e+motivaci\%C3\%B3n\&hl=es\&sa=X\&ved=0ahUKEwjz36Wjmt_eAhUNZKwKHR33Cy8Q6AEISzA

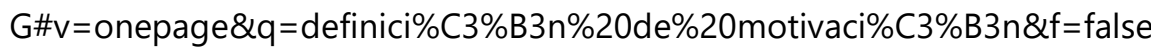

Ausbel, N. (1983). Psicología Educativa: Un punto de vista cognoscitivo. Trillas $2^{\circ} \mathrm{ed}$.

Balongo González, E., \& Mérida Serrano, R. (2016). El clima de aula en los proyectos de trabajo. Crear ambientes de aprendizaje para incluir la diversidad infantil. Perfiles Educativos, 38(152),146162. [fecha de Consulta 12 de Diciembre de 2019]. ISSN: 0185-2698. Disponible en: https://www.redalyc.org/articulo.oa?id=132/13244824009

Busquets, T., Silva, M., \& Larrosa, P. (2016). Reflexiones sobre el aprendizaje de las ciencias naturales: Nuevas aproximaciones y desafíos. Estudios pedagógicos, 42(especial), 117-135. https://dx.doi.org/10.4067/S0718-07052016000300010

Cardona Buitrago, F. (2013). Las prácticas de laboratorio como estrategia didáctica [Trabajo de grado]. Universidad del Valle, Colombia. Recuperado de: http://bibliotecadigital.univalle.edu.co/bitstream/10893/6772/1/CD-0395428.pdf

Díaz Barriga, F., \& Hernández Rojas, G. (1999). Estrategias docentes para el aprendizaje significativo, una interpretación constructivista. Mc Graw-Hill. Recuperado de: http://dip.una.edu.ve/mpe/025disenoinstruccional/lecturas/Unidad_III/EstratDocParaUnAprend Signif.pdf

Díaz Barriga, A. (2013). Secuencias de aprendizaje: ¿Un problema del enfoque de competencias o un reencuentro con perspectivas didácticas? Profesorado. Revista de Currículum y Formación de Profesorado, 17(3),11-33. Recuperado de: https://www.redalyc.org/articulo.oa?id=567/56729527002

Elliott, J. (2000). El cambio educativo desde la investigación-acción. Madrid: Morata.

Elliott, J. (2010). La investigación acción en educación. Madrid: Morata. 
Fierro, C., Fortoul, B., y Rosas, L. (2000). Transformando la práctica docente, una propuesta basada en la investigación-acción. México: Paidós.

Flores Morales, J. (2013). Actividades contextualizadas: una aproximación opción metodológica para fomentar la verbalización estudiantil. Revista Científica de FAREM-Estelí. Medio ambiente, tecnología y desarrollo humano, 7(2),1-15. Recuperado de: http://repositorio.unan.edu.ni/5906/3/95-355-1-PB.pdf

García García, M. (2016) Estrategias didácticas para fomentar la participación de los estudiantes en la asignatura de orientación educativa [Trabajo de grado]. Universidad Autónoma del Estado de México, México.

Giovannone, P. M. (2011) La gestión de la motivación organizacional con el enfoque de la teoría de Herzberg Un estudio empírico [Trabajo de grado]. Universidad Nacional de La Plata, Argentina. Recuperado de: http://hdl.handle.net/10915/22438

Goetz, J., \& LeCompte, M. (1988). Etnografía y diseño cualitativo en investigación educativa. Madrid: Morata. Recuperado de: https://upeldem.files.wordpress.com/2018/03/libro-etnografc3ada-ydisec3b1o-cualitativo-en-investigacic3b3n-educatica-j-p-goetz-y-m-d-lecompte.pdf

ITESM (s/a) Las estrategias y técnicas didácticas en el rediseño. El estudio de casos como técnica didáctica. Dirección de Investigación y Desarrollo Educativo Vicerrectoría Académica. http://sitios.itesm.mx/va/dide2/tecnicas_didacticas/casos/casos.pdf

Latorre, A. (2005). La investigación-acción, conocer y cambiar la práctica educativa. Graó $5^{a}$ ed.

López Sánchez, M., Ramos López, L., Pato López, O., \& López Álvarez, S. (2012). La simulación clínica como herramienta de aprendizaje. Cirugía Mayor Ambulatoria, 18(1),25-29. Recuperado de: http://www.asecma.org/Documentos/Articulos/05_18_1_FC_Lo\%C2\%A6\%C3\%BCpez.pdf

Martínez Izaguirre, M., Yániz Álvarez, C., \& Villardón Gallego, L. (2017). Competencias profesionales del profesorado de educación obligatoria. Revista Iberoamericana De Educación, 74, 171-192. Recuperado de: https://rieoei.org/RIE/article/view/613/1155

Mateo Soriano, M. (2001). La motivación, pilar básico de todo tipo de esfuerzo. Revista de relaciones laborales, 9, 163-184. ISSN: 1133-3189. Recuperado de: https://dialnet.unirioja.es/descarga/articulo/209932.pdf

Mckernan, J. (2001). Investigación-acción y currículum. Madrid: Morata.

Naranjo, M. (2009). Motivación: perspectivas teóricas y algunas consideraciones de su importancia en el ámbito educativo. Revista Educación, 33(2),153-170. https://www.redalyc.org/articulo.oa?id=440/44012058010

Navarrete Sánchez, E., \& Farfán García, M. (2016). Investigación, acción de la reflexión a la práctica educativa. México: Colofón.

Orkaizagirre Gómora, A., Amezcua, M., Huércanos Esparza, I., \& Arroyo Rodríguez, A. (2014). El Estudio de casos, un instrumento de aprendizaje en la Relación de Cuidado. Index de Enfermería, 23(4), 244-249. DOI: 10.4321/S1132-12962014000300011 
Otero Chambean. J. (s/a). Breve manual para elaborar Secuencia Didáctica. Disponible en: http://educacionyculturaaz.com/wp-content/uploads/2014/05/Breve-Manual-para-secuenciasdidacticas.pdf

Quintero, J., Zuluaga C., \& López, M. (2003). La investigación-acción mejora la planeación de clases en lengua extranjera. Íkala, revista de lenguaje y cultura, 8(14),39-56. ISSN: 0123-3432. Recuperado de: https://www.redalyc.org/articulo.oa?id=2550/255026028002

Rioseco, G., \& Romero, R. (2000). La contextualización de la enseñanza de la ciencia como elemento facilitador del aprendizaje significativo. Revista Paideia, 10(24), 253-262. Recuperado de: https://www.oei.es/historico/equidad/rioseco3.PDF

Rodríguez, M. L. (2007). Aplicación del modelo de evaluación CIPP al programa de ciencias de una escuela del distrito escolar de Juana Díaz [TRabajo de grado]. Universidad Interamericana de Puerto Rico, Puerto Rico. Recuperado de: http://ponce.inter.edu/cai/Tesis Graduado/mrodriguez/index.pdf

Rodríguez, L. (2016). La investigación-acción como instrumento de evaluación de la propia práctica docente. IE Revista de Investigación Educativa de la REDIECH, 7(12),52-59. Recuperado de: https://www.redalyc.org/articulo.oa?id=5216/521653208005

Salmerón, L. (2013). Actividades que promueven la transferencia de los aprendizajes: una revisión de la literatura. Revista de Educación, 20013, 1-14. Recuperado de: https://core.ac.uk/download/pdf/71022918.pdf

Torres Villareal, L., Iregui Parra, P., \& Senior Serrano, S. (2015). El interés público en América Latina: Reflexiones desde la educación legal, clínica y el trabajo ProBono. Universidad del Rosario, Bogotá. Recuperado de: https://books.google.es/books?id=jKMyDwAAQBAJ\&printsec=frontcover\&dq=El+inter\%C3\% A9s+p\%C3\%BAblico+en+Am\%C3\%A9rica+Latina:+Reflexiones+desde+la+educaci\%C3\%B3n+ legal, +cl\%C3\%ADnica +y+el +trabajo +probono\&hl=es419\&sa $=X \& v e d=0$ ahUKEwjm vSZo4HnAhVhx4UKHYWkCKwQ6AEIKTAA\#v $=$ onepage \&q $=E \mid \% 2$ 0inter\%C3\%A9s\%20p\%C3\%BAblico\%20en\%20Am\%C3\%A9rica\%20Latina\%3A\%20Reflexiones\% 20desde\%20la\%20educaci\%C3\%B3n\%20legal\%2C\%20cl\%C3\%ADnica\%20y\%20el\%20trabajo\% 20probono\&f=false

Trianes Torres, V. (2012). Psicología del desarrollo y de la educación. Pirámide. 